\title{
DISPONIBILIDADE NO SOLO, ESTADO NUTRICIONAL E RECOMENDAÇÃO DE ENXOFRE PARA O ARROZ IRRIGADO $^{(1)}$
}

\author{
Felipe de Campos Carmona ${ }^{(2)}$, Osmar Conte ${ }^{(3)}$, Thiago Isquierdo Fraga ${ }^{(4)}$, \\ Thiago Barros $^{(5)}$, Edward Pulver ${ }^{(6)}$ \& Ibanor Anghinoni ${ }^{(7)}$
}

\begin{abstract}
RESUMO
A região da Depressão Central do Rio Grande do Sul caracteriza-se por apresentar vastas áreas com solos arenosos e intensivamente cultivados com arroz irrigado por várias décadas. Atualmente, os altos índices de produtividade obtidos pela cultura, associados aos baixos teores de matéria orgânica dos solos da região e às práticas de fertilização utilizadas, constituem condições favoráveis à manifestação de deficiência de S. Os objetivos deste trabalho foram determinar a distribuição do S disponível no perfil do solo e fazer recomendações desse nutriente para o arroz irrigado a partir do estado nutricional, da curva de calibração e da resposta da cultura à adição de doses de adubo sulfatado. $O$ trabalho foi realizado em 12 locais, em solos de várzea da região da Depressão Central, nas safras 2004/ 2005 e 2005/2006. Os teores de $\mathrm{S}$ disponível no perfil do solo não se relacionam com os teores de matéria orgânica e de argila. A concentração de $\mathrm{S}$ no tecido do arroz aumenta com a disponibilidade desse nutriente no solo e com as doses de adubo aplicadas. $O$ teor de $\mathrm{S}$ nas plantas de arroz não atinge a faixa de suficiência pela interpretação do seu estado nutricional em uso no Brasil. O teor crítico de $S$ determinado para solos da Depressão Central do Rio Grande do Sul é superior ao
\end{abstract}

\footnotetext{
(1) Parte da Dissertação de Mestrado apresentada pelo primeiro autor ao Programa de Pós-Graduação em Ciência do Solo, Universidade Federal do Rio Grande do Sul - UFRGS. Recebido para publicação em setembro de 2007 e aprovado em fevereiro de 2009.

(2) Engenheiro-Agrônomo, Doutorando do Programa de Pós-Graduação em Ciência do Solo, Universidade Federal do Rio Grande do Sul - UFRGS. Av. Bento Gonçalves 7712, CEP 91540-000 Porto Alegre (RS). Bolsista CAPES. E-mail: felipecamposcarmona@hotmail.com

(3) Engenheiro-Agrônomo, Doutorando do Programa de Pós-Graduação em Ciência do Solo - UFRGS. E-mail: conte_osmar@hotmail.com

${ }^{(4)}$ Acadêmico do Curso de Agronomia - UFRGS. E-mail: isquierdofraga@yahoo.com.br

(5) Engenheiro-Agrônomo. Grão Inteiro Sistemas de Gestão Agropecuária. Av. Nova York 211/502, CEP 90550-070 Porto Alegre (RS). E-mail: thiagopoabr@hotmail.com

(6) Biólogo, Consultor do Centro Internacional de Agricultura Tropical - CIAT. Recta Cali - Palmira, km 17, A.A. 6713, Cali, Colômbia. E-mail: edwardpulver@yahoo.com

${ }^{(7)}$ Professor Adjunto, Programa de Pós-Graduação em Ciência do Solo, UFRGS. Bolsista CNPq. E-mail: ibanghi@ufrgs.br
} 


\begin{abstract}
utilizado no sul do Brasil. As doses de máxima eficiência técnica (36 kg ha-1) e econômica (26 kg ha-1) para o arroz irrigado em solos com teor abaixo do crítico resultam em ganho similar no rendimento de grãos.
\end{abstract}

Termos de indexação: Oryza sativa, sulfato, curva de calibração, teor crítico.

\author{
SUMMARY: SOIL AVAILABILITY, NUTRITIONAL STATUS AND SULFUR \\ RECOMMENDATION FOR FLOODED RICE
}

\begin{abstract}
The Depressão Central region in the State of Rio Grande do Sul, Brazil, is characterized by large areas of sandy soil that were intensively cultivated with flooded rice in the last decades. Currently, the high rice yields, along with the low organic matter content of these soils and the fertilization practices are favorable conditions for the appearance of sulfur (S) deficiency. With the purpose of determining sulfur distribution in the soil profile and recommending levels of this element for flooded rice based on the calibration curve and crop response to sulfur fertilizer, a study was carried out at 12 sites in the Depressão Central region during the 2004/ 2005 and 2005/2006 growing seasons. Sulfur availability in the soil profile did not correlate with organic matter and clay content. Sulfur content in plant tissue increased with soil sulfur availability and with soil-applied sulfur rates, but did not reach the sufficiency range determined by the interpretation system used in Brazil. The critical content determined in the soils of the Depressão Central in the State of Rio Grande do Sul is higher than the one currently used in Southern Brazil. The sulfur rates for the maximum technical efficiency (36 $\left.\mathrm{kg} \mathrm{ha}^{-1}\right)$ and economic efficiency $\left(26 \mathrm{~kg} \mathrm{ha}^{-1}\right)$ for flooded rice on soils with sulfur availability below the critical level resulted in similar grain yields.
\end{abstract}

Index terms: Oryza sativa, sulfate, calibration curve, critical content. |

\section{INTRODUÇÃO}

A produção de arroz no Brasil é originária, principalmente, das lavouras irrigadas do Rio Grande do Sul, que ocupam cerca de $31 \%$ da área cultivada e contribuem com aproximadamente $53 \%$ da produção nacional desse cereal (CONAB, 2007). Nesse contexto, a região arrozeira da Depressão Central é uma das mais importantes, pois concentra cerca de $16 \%$ de toda a produção desse cereal e $50 \%$ dos produtores do Estado (IRGA, 2008). Predominam, nas áreas aptas ao cultivo de arroz irrigado nessa região, os Planossolos Hidromórficos e os Gleissolos Háplicos (Pinto et al., 2004; Streck et al., 2008), com predominância dos Gleissolos nas cotas mais baixas junto aos rios Jacuí, Vacacaí, Vacacaí-Mirim e Santa Maria. Diferentemente de outras regiões, como a Fronteira Oeste e a Campanha, onde o cultivo de arroz é mais recente e há alternância de áreas com pecuária e soja, na Depressão Central muitas das terras utilizadas para a produção de arroz não passam por qualquer tipo de rotação de culturas há anos e, em muitos casos, sequer ocorre a prática de pousio.

Atualmente, apesar de a maioria dos cultivares disponíveis apresentar potencial produtivo superior a 10 t ha-1, algumas áreas do Rio Grande do Sul mostram limitações ao cultivo do arroz irrigado, seja pelo uso intensivo da terra, seja pelas características naturais de baixa fertilidade do solo. Em virtude desse panorama, o Projeto de Transferência de Tecnologia para Altas Produtividades, resultado de convênio firmado entre o Instituto Rio Grandense do Arroz (IRGA), Fondo Latino Americano para Arroz de Riego (FLAR) e Common Fund for Commodities (CFC), foi implementado no Estado, no ano agrícola 2003/2004, com o objetivo de aumentar a média de produtividade da cultura. Neste trabalho, ao contrário dos resultados obtidos nas regiões Campanha e Fronteira Oeste, onde as produtividades médias foram superiores a $9,0 \mathrm{tha}^{-1}$, algumas lavouras da Depressão Central, especialmente nos municípios de Restinga Seca, Rio Pardo e Agudo, não atingiram as produtividades almejadas. Mesmo com a adoção de práticas adequadas de manejo, o rendimento médio não superou a marca de 7,5 t ha ${ }^{-1}$ (Pulver \& Carmona, 2004). Nos locais de menor rendimento, foi verificado que as plantas apresentavam visível amarelecimento, porte baixo e perfilhamento reduzido, não condizente com o seu estádio de desenvolvimento, mesmo com a aplicação de doses elevadas de N (até $200 \mathrm{~kg} \mathrm{ha}^{-1} \mathrm{de} \mathrm{N}$ ). Os baixos teores de argila e de matéria orgânica do solo nessas áreas, associados com a realização de cortes profundos para o nivelamento dos quadros, também indicavam a possibilidade de o $\mathrm{S}$ ter sido o fator limitante. Isso porque, assim como em solos oxidados, a quantidade 
de $\mathrm{S}_{-} \mathrm{SO}_{4}{ }^{2-}$ disponível em solos alagados está diretamente relacionada à textura e ao teor de matéria orgânica. Além disso, como a manutenção da lâmina d'água nas lavouras pode ser superior a 150 dias por ano, especialmente no sistema de cultivo prégerminado, a redução do sulfato pode causar deficiência de $\mathrm{S}$ em solos com baixa disponibilidade desse elemento, devido às perdas por volatilização de $\mathrm{H}_{2} \mathrm{~S}$ (Lefroy et al., 2005).

Nesse contexto, o teor de argila do solo é um dos fatores que determina a manutenção do $\mathrm{S}_{-} \mathrm{SO}_{4}{ }^{2-}$ ao alcance das raízes das plantas de arroz. Os solos argilosos têm maior capacidade de retenção de $\mathrm{S}_{-} \mathrm{SO}_{4}{ }^{2-}$ porque possuem normalmente teores elevados de óxidos de $\mathrm{Fe}$, tornando mais lento o seu movimento no perfil. Em solos arenosos, o S-SO ${ }_{4}^{2-}$ tende a se deslocar mais rapidamente para os horizontes subsuperficiais, por lixiviação (Ensminger, 1954). Bissani (1985) constatou, em solos de várzea do Rio Grande do Sul, com menos de $200 \mathrm{~g} \mathrm{~kg}^{-1}$ de argila,

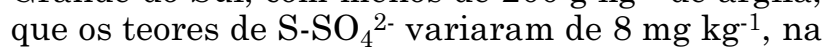
camada de solo de $0-20 \mathrm{~cm}$, a $13 \mathrm{mg} \mathrm{kg}^{-1}$, na camada de $20-40 \mathrm{~cm}$, e estavam relacionados com o teor de argila, que aumentava com a profundidade de amostragem. Entretanto, essa dinâmica pode ser diferente quando esses solos são utilizados para o cultivo do arroz irrigado, pois, mesmo se drenados superficialmente, podem manter uma camada subsuperficial ainda em estado de anaerobiose, com consequente volatilização e perda de $\mathbf{S}$ do sistema (Jordan \& Ensminger, 1958), fazendo com que os teores desse nutriente em camadas mais profundas sejam menores, como verificado por Beaton \& White (1997) em camada abaixo de $20 \mathrm{~cm}$ de solos das Filipinas.

Estudos relacionados à necessidade de $\mathrm{S}$ para o arroz irrigado são realizados com mais frequência em países de clima tropical, cujos solos são intemperizados e altamente consumidores desse cereal, como os do sul asiático. Em Bangladesh, por exemplo, a aplicação de $20 \mathrm{~kg} \mathrm{ha}{ }^{-1}$ de $\mathrm{S}_{-} \mathrm{SO}_{4}{ }^{2-}$ resultou num acréscimo em produtividade de 0,5 a $1,0 \mathrm{t} \mathrm{ha}^{-1}$ (Beaton \& White, 1997). Já na Índia, em estudo conduzido na província de Bengal do Oeste, Mandal et al. (1997) concluíram que, independentemente da fonte, doses de $20 \mathrm{~kg} \mathrm{ha}^{-1}$ de $\mathrm{S}_{-} \mathrm{SO}_{4}{ }^{2-}$ incrementaram as produtividades entre 20 e $30 \%$. Resultados semelhantes foram obtidos mais recentemente na Colômbia (Medina, 2003; Riobueno, 2003), onde a aplicação de S gerou acréscimos de até 2,3 $\mathrm{t} \mathrm{ha}^{-1}$. Nos Estados Unidos, em solos deficientes desse nutriente, é recomendada a aplicação de $100 \mathrm{~kg} \mathrm{ha}^{-1}$ de sulfato de amônio (Wilson Jr. et al., 2006), o que corresponde à adição de $24 \mathrm{~kg} \mathrm{ha}^{-1} \mathrm{de} \mathrm{S}$.

Este trabalho teve como objetivos determinar a distribuição do S disponível no perfil do solo e estabelecer recomendações desse nutriente para o arroz irrigado a partir da evolução do estado nutricional, da obtenção da curva de calibração e da resposta da cultura à adição de doses de adubo sulfatado.

\section{MATERIAL E MÉTODOS}

O trabalho foi realizado no ano agrícola 2003/2004, na região arrozeira da Depressão Central do RS, onde se verificou resposta do arroz irrigado à adição de $\mathrm{S}$ em lavouras comerciais de Restinga Seca, Agudo e Rio Pardo (Pulver \& Carmona, 2004). No ano agrícola 2004/2005, o trabalho visou basicamente determinar a resposta da cultura à adição de $\mathrm{S}$ em solos com diferentes teores de S-SO ${ }_{4}^{2-}$. Já em 2005/2006, houve maior aprofundamento, com a avaliação dos teores desse nutriente em camadas de solo, da evolução dos seus teores na planta e da resposta da cultura à aplicação de doses de adubo sulfatado.

A escolha dos locais para instalação dos experimentos em ambas as safras foi feita com base em uma amostragem prévia dos solos de 41 lavouras de produtores envolvidos no Projeto de Transferência de Tecnologia antes referido em diversos municípios da região arrozeira da Depressão Central do RS. Foram selecionadas áreas que contemplassem os requisitos para a implantação do trabalho, como facilidade e independência de irrigação, além de ausência de taipas e de cortes profundos (superiores a $20 \mathrm{~cm}$ ), quando do nivelamento dos quadros. Nos locais com potencial para o desenvolvimento do trabalho, foram retiradas, com trado de rosca, 15 amostras simples de solo na camada de 0-20 cm em áreas de cerca de 1 ha. A partir dos resultados das análises, foram selecionados 12 locais, seis em cada ano, que contemplassem quatro faixas de teores de $\mathrm{S}_{-} \mathrm{SO}_{4}{ }^{2-}$ disponível (extrator $\left.\mathrm{Ca}\left(\mathrm{H}_{2} \mathrm{PO}_{4}\right)_{2}, 500 \mathrm{mg} \mathrm{L}^{-1} \mathrm{de} \mathrm{P}\right): 0$ $5,0 \mathrm{mg} \mathrm{dm}^{-3} ; 5,1-10,0 \mathrm{mg} \mathrm{dm}^{-3} ; 10,1-15,0 \mathrm{mg} \mathrm{dm}^{-3} ; \mathrm{e}$ mais de $15 \mathrm{mg} \mathrm{dm}^{-3}$.

Os experimentos foram conduzidos em 11 propriedades rurais e um na subestação Experimental do IRGA, na barragem do Capané, em Cachoeira do Sul (Quadro 1). Dez experimentos foram implantados em Gleissolo Háplico (Embrapa, 1999) e dois, um na barragem do Capané e outro em Minas do Leão, foram em Planossolo Hidromórfico (Embrapa, 1999). Utilizaram-se os cultivares de ciclo precoce IRGA 422 CL em 11 áreas, pela alta infestação de arroz-vermelho, e IRGA 417 em uma das áreas (Rio Pardo I), sem infestação dessa invasora. Todas as áreas experimentais foram semeadas com semeadora, em linha, no espaçamento de $17 \mathrm{~cm}$ entre linhas. A época de semeadura, nos diversos locais, deu-se no intervalo entre 15 de outubro e 15 de novembro, de acordo, portanto, com o Zoneamento Agroclimático de Arroz Irrigado (SOSBAI, 2005). A densidade de semeadura foi de $80 \mathrm{~kg} \mathrm{ha}^{-1}$, e as sementes receberam tratamento com inseticida à base de fipronil para prevenir a infestação de Oryzophagus oryzae, a bicheira-da-raiz (Pulver et al., 2005). O controle de plantas daninhas foi realizado em pós-emergência, no estádio de desenvolvimento V3-V4 (três a quatro folhas totalmente desenvolvidas), segundo escala de Counce et al. (2000). 
Quadro 1. Características químicas e teor de argila dos solos coletados antes da instalação dos experimentos em diferentes locais, nas safras 2004/2005 e 2005/2006

\begin{tabular}{|c|c|c|c|c|c|c|}
\hline Local & pH $\mathrm{H}_{2} \mathrm{O}(1: 1)$ & Argila & Matéria orgânica & P disponível ${ }^{(3)}$ & K disponível $^{(3)}$ & $\mathrm{S}-\mathrm{SO}_{4}{ }^{(4)}$ \\
\hline & & $-\xi$ & $\mathrm{kg}^{-1}$ & 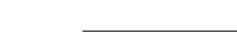 & $\mathrm{mg} \mathrm{dm}{ }^{-3}$ & - \\
\hline 1. Restinga Seca I ${ }^{(2)}$ & 4,7 & 100 & 13 & 18 & 88 & 2,0 \\
\hline 2. Cachoeira do Sul I $\mathrm{I}^{(1)}$ & 4,9 & 120 & 6 & 29 & 104 & 4,5 \\
\hline 3. Minas do Leão(2) & 4,4 & 100 & 11 & 6 & 89 & 6,4 \\
\hline 4. Restinga Seca II ${ }^{(2)}$ & 4,9 & 90 & 9 & 9 & 50 & 6,6 \\
\hline 5. Rio Pardo $I^{(1)}$ & 5,3 & 150 & 16 & 21 & 104 & 6,7 \\
\hline 6. Rio Pardo II ${ }^{(1)}$ & 5,9 & 130 & 15 & 29 & 87 & 7,3 \\
\hline 7. Restinga Seca III ${ }^{(1)}$ & 4,4 & 320 & 15 & 5 & 74 & 9,4 \\
\hline 8. Restinga Seca IV ${ }^{(1)}$ & 4,4 & 160 & 11 & 6 & 83 & 9,6 \\
\hline 9. Restinga Seca V ${ }^{(2)}$ & 4,7 & 90 & 8 & 7 & 68 & 13,4 \\
\hline 10. Cachoeira do Sul II ${ }^{(2)}$ & 4,5 & 230 & 14 & 4 & 72 & 15,6 \\
\hline 11. Restinga Seca VI ${ }^{(2)}$ & 4,3 & 290 & 32 & 4 & 97 & 16,5 \\
\hline 12. Cachoeira do Sul III ${ }^{(1)}$ & 4,2 & 280 & 15 & 13 & 65 & 19,0 \\
\hline
\end{tabular}

${ }^{(1)}$ Safra 2004/2005. ${ }^{(2)}$ Safra 2005/2006. ${ }^{(3)}$ Mehlich-1. ${ }^{(4)}$ Extrator Ca($\left(\mathrm{H}_{2} \mathrm{PO}_{4}\right)_{2}, 500 \mathrm{mg} \mathrm{L}{ }^{-1}$ de P.

A adubação de base foi realizada, em função dos resultados das análises de solo das amostragens coletadas, conforme citado anteriormente. O procedimento seguiu as recomendações do Projeto de Transferência de Tecnologia e visou a obtenção de elevados rendimentos. Foram aplicados, em todos os locais, $50 \mathrm{~kg} \mathrm{ha}^{-1}$ de $\mathrm{P}_{2} \mathrm{O}_{5}$, via MAP, na linha de semeadura, e 80 a $120 \mathrm{~kg} \mathrm{ha}^{-1}$ de $\mathrm{K}_{2} \mathrm{O}$, na forma de $\mathrm{KCl}$, aplicados a lanço em cobertura. Essas recomendações são similares às atuais (SOSBAI, 2007) para alta produtividade do arroz irrigado.

Os tratamentos foram aplicados no início do perfilhamento (V3-V4) e constaram da aplicação manual e a lanço, sem incorporação ao solo, de 0,10 , 20,40 e $80 \mathrm{~kg} \mathrm{ha}^{-1} \mathrm{de} \mathrm{S}$, na forma de sulfato de amônio. A dose de N aplicada, juntamente com o sulfato de amônio, foi de $210 \mathrm{~kg} \mathrm{ha}^{-1}$ no ano agrícola 2004/2005

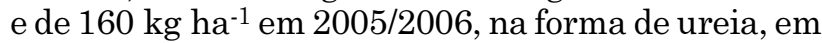
complementação ao $\mathrm{N}$ aplicado como sulfato de amônio nos tratamentos e como MAP na adubação de base.

Imediatamente antes da aplicação dos tratamentos, foram retiradas amostras de solo para a caracterização das áreas experimentais: (a) com trado de rosca, na camada de 0-20 cm, no ano agrícola 2004/2005 e (b) com trado calador, nas camadas de 0-10, 10-20 e 20$30 \mathrm{~cm}$, no agrícola 2005/2006. Em ambos os anos, as amostras foram compostas de 15 amostras simples por bloco, cujos resultados médios foram ordenados conforme o teor de $\mathrm{S}$ no solo (Quadro 1).

No ano agrícola 2004/2005, os experimentos foram conduzidos em parcelas de $24 \mathrm{~m}^{2}(6 \times 4 \mathrm{~m})$, dispostas em blocos ao acaso, com espaçamento de 1,5 m entre as parcelas e três repetições. No ano agrícola 2005/ 2006 , as parcelas foram de $75 \mathrm{~m}^{2}(10 \times 7,5 \mathrm{~m})$, delineadas em blocos ao acaso, com espaçamento de $3 \mathrm{~m}$ entre parcelas, com quatro repetições. Os trabalhos, nos dois anos, foram realizados em locais diferentes, sem histórico de fertilização recente com algum adubo que contivesse S. A entrada d'água ocorreu, no máximo, $8 \mathrm{~h}$ depois dos tratamentos, mantendo-se uma lâmina de água de 5 a $10 \mathrm{~cm}$ até a fase de maturação fisiológica da cultura.

No ano agrícola 2005/2006, além da avaliação do rendimento de grãos, também foram feitas coletas de plantas de arroz para a determinação dos teores de $\mathrm{S}$ no tecido (método da digestão ácida - Tedesco et al., 1995) e de S absorvido aos 28 dias após o início da irrigação (DAI), período suficiente para a disponibilização do S adicionado como fertilizante (Vitti et al., 1986). Em Cachoeira do Sul II, a coleta foi retardada em sete dias, em função do estabelecimento inicial irregular da cultura. Plantas de duas linhas de $50 \mathrm{~cm}$ de arroz, situadas a uma distância de $50 \mathrm{~cm}$ das bordas das parcelas, foram cortadas com foice, rente à superfície do solo, retirando-se um total de 50 plantas por parcela. Por ocasião do pleno florescimento das lavouras (estádio R3), foi feita a coleta de 30 folhas-bandeiras por parcela, para determinação do teor de $\mathrm{S}$ no tecido. O material vegetal foi seco em estufa com ventilação forçada de ar, em temperatura de aproximadamente $50^{\circ} \mathrm{C}$, e moído para análise do teor de $\mathrm{S}$ (Tedesco et al., 1995).

Ao final do ciclo da cultura, no ano agrícola 2004/ 2005, foi feita a colheita manual dos ensaios em uma área de $12 \mathrm{~m}^{2}(3 \times 4 \mathrm{~m})$ em cada parcela. No ano agrícola seguinte, a área colhida foi de $5 \mathrm{~m}^{2}(2 \times 2,5 \mathrm{~m})$. O material colhido foi trilhado em trilhadora estacionária. Os grãos foram limpos com peneira e pesados. O peso foi corrigido para a umidade de $13 \%$, para o cálculo de rendimento de grãos. Após secagem em estufa com ventilação forçada, foi feita análise do teor de $\mathrm{S}$ nos grãos pelo método de digestão ácida (Tedesco et al., 1995) e calculada a quantidade exportada. 


\section{RESULTADOS E DISCUSSÃO}

Teores de enxofre, de matéria orgânica e de argila no perfil do solo

Apesar da variabilidade observada nesses atributos, os teores são baixos na maioria dos solos e podem ser atribuídos, em adição à sua condição natural, ao cultivo intensivo e às práticas de manejo antes utilizadas nos locais dos experimentos, especialmente ao manejo da lâmina d'água nas lavouras (Marcolin et al., 2005).

Considerando-se que o teor de argila do solo tende a aumentar em profundidade (Quadro 2), com uma única exceção (Restinga Seca V), esperava-se, a exemplo do encontrado por Bissani (1985), que os teores de $\mathrm{S}_{-} \mathrm{SO}_{4}{ }^{2-}$ também aumentassem. Isso porque esse íon é passível de lixiviação do horizonte superficial do solo, adsorvendo-se às cargas positivas de óxidos de $\mathrm{Fe}$ e $\mathrm{Al}$ em horizontes subsuperficiais (Hingston et al., 1972). Entretanto, neste estudo, essa relação não ocorreu $\left(r=0,50^{\mathrm{NS}}\right)$, pois, na maioria dos locais, o teor de $\mathrm{S}_{-} \mathrm{SO}_{4}{ }^{2-}$ na camada de $20-30 \mathrm{~cm}$ foi inferior aos valores das camadas de $0-10$ e $10-20 \mathrm{~cm}$. Por se tratar de solos de várzea, a camada de $20-30 \mathrm{~cm}$ passa por períodos de redução mais prolongados do que os da camada de 0-20 cm, em especial durante o inverno e a primavera, estações tipicamente chuvosas no Rio Grande do Sul. É provável que, pelo maior período de saturação na camada de solo de $20-30 \mathrm{~cm}$, o ambiente de redução do solo favoreça a conversão do $\mathrm{S}_{-} \mathrm{SO}_{4}{ }^{2-}$ lixiviado das camadas superiores em $\mathrm{H}_{2} \mathrm{~S}$ (Chaitep et al., 1994; Lefroy et al., 2005). Apesar de a maior parte do $\mathrm{S}$ do solo (75 a $99 \%$ ) ser proveniente da fração orgânica (Bissani et al., 2008) e das tendências apresentadas (Quadro 2), a relação entre eles foi baixa $\left(\mathrm{r}=0,54^{\mathrm{NS}}\right)$, provavelmente pelo uso intensivo e pelo tempo de cultivo do solo com arroz irrigado, além da possibilidade de suprimento de S por outras fontes, como a atmosférica (Osório Filho, 2007).

\section{Absorção e exportação de enxofre pelo arroz}

Os resultados dessas avaliações referem-se à safra 2005/2006 e são oriundos de seis locais. Apenas nos solos com os teores mais baixos de $\mathrm{S}-\mathrm{SO}_{4}{ }^{2-}$ ( $\leq 6,4 \mathrm{mg} \mathrm{dm}^{-3}$ ), houve aumento (ajuste quadrático) na sua absorção pelas plantas de arroz com a aplicação de doses crescentes de adubo sulfatado (Figura 1). Nas outras situações, mesmo com as tendências de aumento apresentadas, não houve significância no ajuste $(p>0,05)$ das regressões obtidas. O estabelecimento das plantas em Restinga Seca VI (Figura 1f) foi o mais lento na comparação com os demais, devido, provavelmente, à textura mais argilosa do solo, o que, pelas condições de umidade, causou prejuízo à emergência das plântulas. Apesar disso, nesse local e em Cachoeira do Sul II (Figura 1e), cujos solos apresentaram os maiores teores de $\mathrm{S}(16,5$ e $15,6 \mathrm{mg} \mathrm{dm}^{-3}$, respectivamente - Quadro 3), houve maior absorção desse nutriente pelas plantas nos tratamentos testemunha.

Quadro 2. Teores de argila, de matéria orgânica e de $\mathrm{S}_{-} \mathrm{SO}_{4}^{-2}$ em camadas dos solos coletados antes da instalação dos experimentos em diferentes locais, na safra 2005/2006

\begin{tabular}{|c|c|c|c|c|}
\hline Local & Camada de solo & Argila & Matéria orgânica & $\mathrm{S}-\mathrm{SO}_{4}{ }^{(1)}$ \\
\hline & $\mathrm{cm}$ & 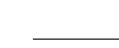 & - & $\mathrm{mg} \mathrm{dm}^{-3}$ \\
\hline 1. Restinga Seca I & $\begin{array}{r}0-10 \\
10-20 \\
20-30\end{array}$ & $\begin{array}{l}100 \\
100 \\
120\end{array}$ & $\begin{array}{r}11 \\
10 \\
5\end{array}$ & $\begin{array}{l}2,2 \\
1,8 \\
1,2\end{array}$ \\
\hline 2. Minas do Leão & $\begin{array}{r}0-10 \\
10-20 \\
20-30\end{array}$ & $\begin{array}{l}100 \\
100 \\
110\end{array}$ & $\begin{array}{r}11 \\
12 \\
6\end{array}$ & $\begin{array}{l}6,2 \\
6,5 \\
5,6\end{array}$ \\
\hline 3. Restinga Seca II & $\begin{array}{r}0-10 \\
10-20 \\
20-30\end{array}$ & $\begin{array}{r}80 \\
100 \\
110\end{array}$ & $\begin{array}{r}11 \\
6 \\
4\end{array}$ & $\begin{array}{l}7,2 \\
6,1 \\
6,3\end{array}$ \\
\hline 4. Restinga Seca V & $\begin{array}{r}0-10 \\
10-20 \\
20-30\end{array}$ & $\begin{array}{r}100 \\
90 \\
90\end{array}$ & $\begin{array}{l}8 \\
6 \\
5\end{array}$ & $\begin{array}{l}11,9 \\
14,8 \\
14,2\end{array}$ \\
\hline 5. Cachoeira do Sul II & $\begin{array}{r}0-10 \\
10-20 \\
20-30\end{array}$ & $\begin{array}{l}210 \\
240 \\
270\end{array}$ & $\begin{array}{r}14 \\
11 \\
9\end{array}$ & $\begin{array}{l}16,7 \\
14,6 \\
10,2\end{array}$ \\
\hline 6. Restinga Seca VI & $\begin{array}{r}0-10 \\
10-20 \\
20-30\end{array}$ & $\begin{array}{l}260 \\
310 \\
350\end{array}$ & $\begin{array}{l}40 \\
33 \\
24\end{array}$ & $\begin{array}{l}16,5 \\
16,5 \\
10,5\end{array}$ \\
\hline
\end{tabular}

\footnotetext{
(1) Extrator $\mathrm{Ca}\left(\mathrm{H}_{2} \mathrm{PO}_{4}\right)_{2}, 500 \mathrm{mg} \mathrm{L}{ }^{-1}$ de $\mathrm{P}$.
} 

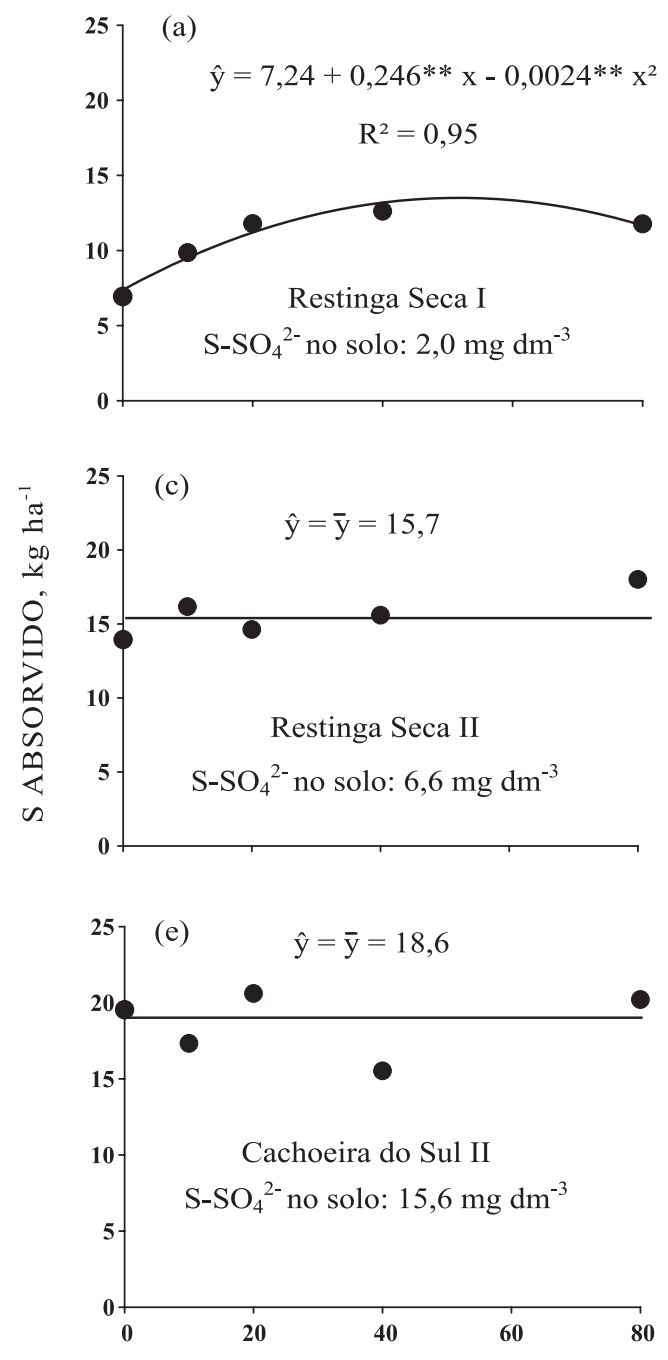
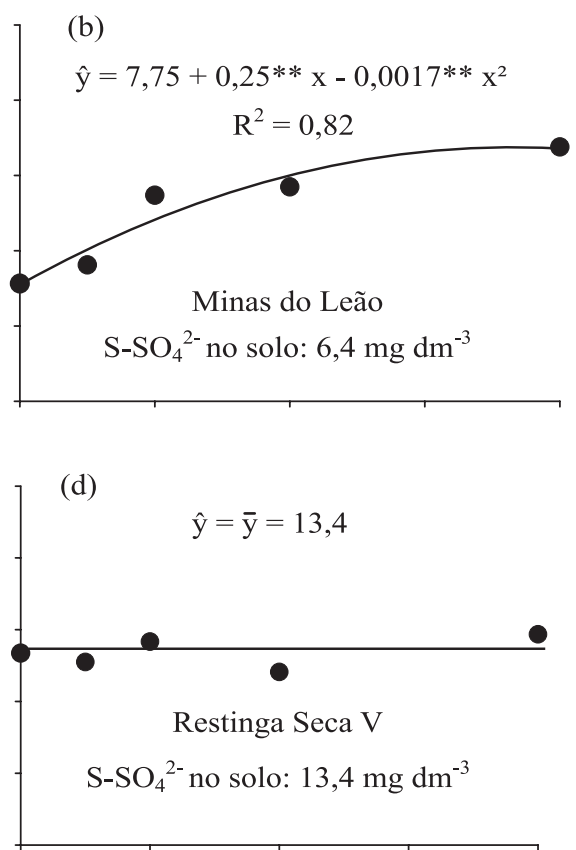

(f)

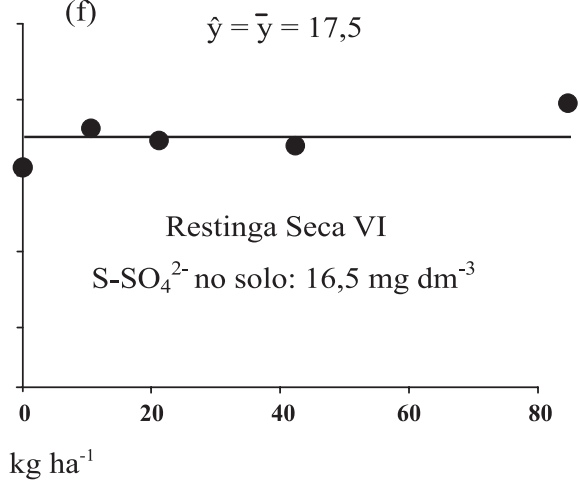

Figura 1. Enxofre absorvido pelo arroz irrigado, em função da aplicação de doses deste elemento em diferentes locais, na safra $2005 / 2006$. **Significativo pelo teste $\mathrm{F}(\mathrm{p}<0,01)$.

As quantidades de $\mathrm{S}$ absorvidas pelo arroz refletem os seus teores no tecido da parte aérea (Quadro 3), uma vez que foram diferenciados (interação significativa; $p<0,05)$ em função dos teores no solo e das doses de adubo sulfatado aplicadas (Quadro 3). Assim, os aumentos no teor do nutriente no tecido da parte aérea ocorreram predominantemente nos solos com teores de $\mathrm{S}$ mais baixos $\left(\leq 6,4 \mathrm{mg} \mathrm{dm}^{-3}\right)$ e até doses intermediárias do adubo (20 e $40 \mathrm{~kg} \mathrm{ha}^{-1}$ de S) no solo com o teor mais baixo $\left(2,0 \mathrm{mg} \mathrm{dm}^{-3}\right)$, onde a resposta à aplicação foi quadrática. Nos demais casos, a resposta foi predominantemente linear. Alam et al. (1985) reportaram aumentos nos teores de $\mathrm{S}$ no tecido do arroz em resposta à adição de até $15 \mathrm{~kg} \mathrm{ha}^{-1} \mathrm{de} \mathrm{S}$, em solos deficientes desse nutriente. Por outro lado, os teores de $\mathrm{S}$ na folha-bandeira variaram menos entre solos e entre as doses desse nutriente aplicadas (Quadro 3), havendo resposta (linear) somente em dois locais, onde os teores no solo estavam entre os mais baixos $\left(2,0\right.$ e $\left.6,6 \mathrm{mg} \mathrm{dm}^{-3}\right)$.
Os teores de S na parte aérea da planta e na folhabandeira aumentaram com os aumentos dos teores de $\mathrm{S}$ disponível no solo, tanto no tratamento testemunha (sem adição de $\mathrm{S}$ ) como naqueles que receberam a aplicação de doses desse nutriente (Quadro 3). Apenas em dois locais (Restinga Seca I e Minas do Leão), que apresentaram os menores teores de $\mathrm{S}_{-} \mathrm{SO}_{4}{ }^{2-}$ no solo (Quadro 1), os teores de $\mathrm{S}$ no tecido vegetal da parte aérea do tratamento testemunha foram inferiores a $1,0 \mathrm{~g} \mathrm{~kg}^{-1}$, teor considerado baixo para a cultura (Wilson Jr. et al., 2006). Segundo esses autores, a planta de arroz bem nutrida de $\mathrm{S}$ deve apresentar teores no tecido de 1,7 e $1,5 \mathrm{~g} \mathrm{~kg}^{-1}$, no perfilhamento e na iniciação do primórdio floral, respectivamente. Sendo isso verdadeiro, na maior parte dos casos deste trabalho, o teor desse nutriente na planta (parte aérea e folha-bandeira) seria considerado médio, e somente no solo com o maior teor de $\mathrm{S}\left(16,5 \mathrm{mg} \mathrm{dm}^{-3}\right.$ - Restinga Seca IV) os teores desse nutriente seriam considerados altos (faixa de 
Quadro 3. Teor de enxofre no tecido da parte aérea e na folha-bandeira no florescimento pleno do arroz irrigado e respectiva equação de regressão em função de doses de adubo aplicadas em solos com diferentes teores de $\mathrm{S}$ disponível

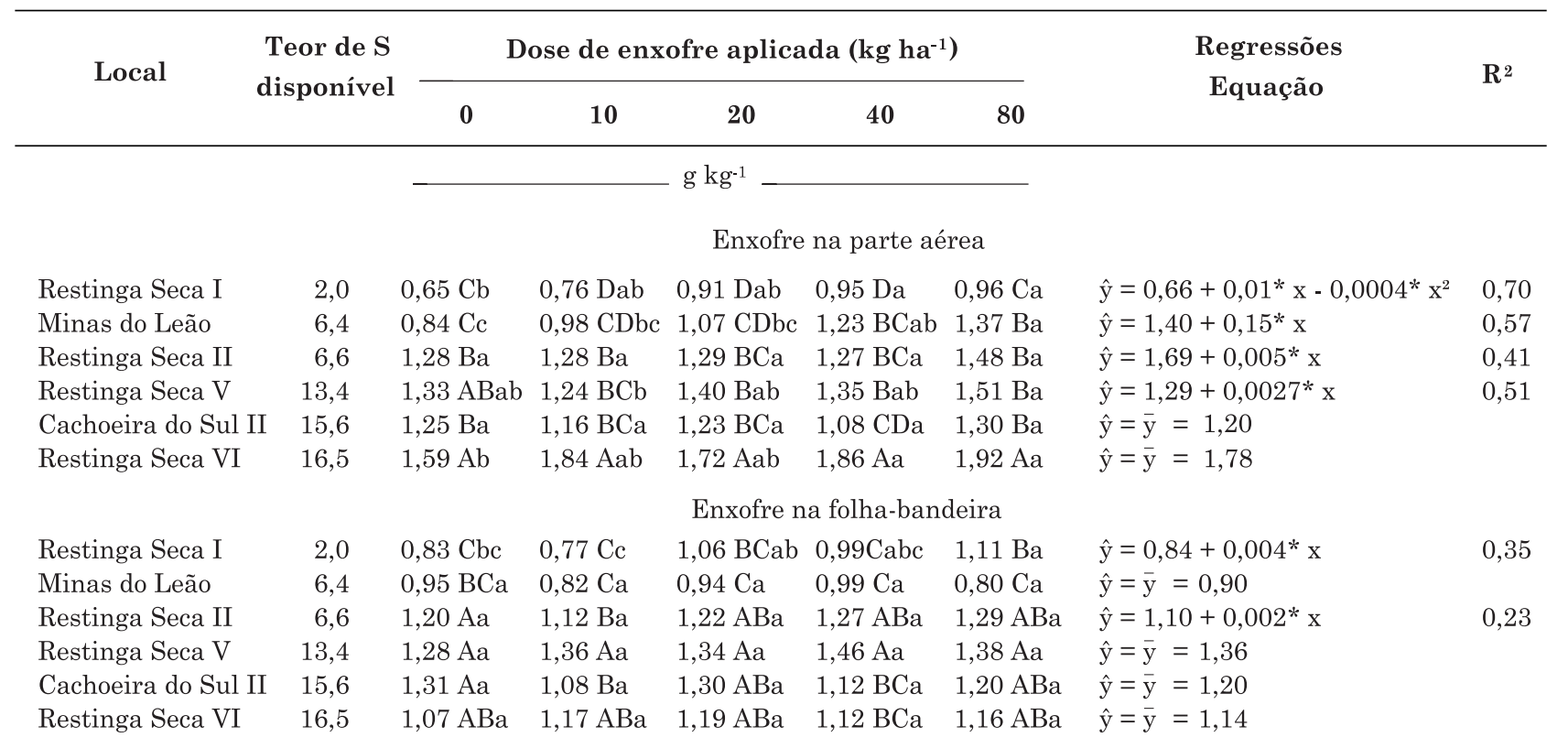

Médias seguidas de mesma letra maiúscula na coluna e minúscula na linha não diferem entre si pelo teste de Tukey a $5 \%$. * significativo pelo teste $\mathrm{F}(\mathrm{p}<0,05)$.

suficiência). Com relação aos teores de S na folhabandeira, em nenhuma situação (Quadro 3) haveria suficiência do nutriente segundo Wilson Jr. et al. (2006) (S > 1,5 g kg-1), Malavolta et al. (1997) (S entre 1,5 e 2,0 $\mathrm{g} \mathrm{kg}^{-1}$ ) e CQFS RS/SC (2004) (Sentre 2,0 e 3,0 g kg1), mesmo com produtividades em torno de $9,0 \mathrm{t} \mathrm{ha}^{-1}$ de grãos (Carmona, 2007). No entanto, considerando o teor crítico de $\mathrm{S}$ na folha-bandeira, estabelecido pelo IRRI (2007) de $1,0 \mathrm{~g} \mathrm{~kg}^{-1}$, e a faixa de suficiência entre 1,0 e $1,5 \mathrm{~g} \mathrm{~kg}^{-1}$, a interpretação dos dados deste trabalho (Quadro 3) indica que o teor de S na folhabandeira somente seria considerado baixo no tratamento testemunha e com a aplicação da dose mais baixa (10 kg ha-1 de $\mathrm{S})$ no solo onde o teor de $\mathrm{S}$ disponível foi o menor (Restinga Seca I - Quadro 1) e onde foi de 6,4 $\mathrm{mg} \mathrm{dm}^{-3}$ (Minas do Leão-Quadro 1), independentemente da adição ou não do fertilizante. Nos demais casos, os teores enquadram-se na faixa de suficiência. Por outro lado, o ponto de equilíbrio nutricional desse nutriente no DRIS (Guindani, 2007) é de $1,8 \mathrm{~g} \mathrm{~kg}^{-1}$ para uma população de alta produtividade $\left(>9,0 \mathrm{t} \mathrm{ha}^{-1}\right)$, para solos de todas as regiões arrozeiras do Rio Grande do Sul, valor bem mais alto do que os encontrados neste trabalho (Quadro 3). Daí a necessidade de se efetuarem estudos para a definição das faixas de interpretação dos resultados de análises de $\mathrm{S}$ no solo e na planta, para a verificação da capacidade de fornecimento desse nutriente pelo solo e do estado nutricional da planta de arroz irrigado, uma vez que, em ambos os casos, há pouco suporte de pesquisa desenvolvida no País para essa cultura.
As quantidades de $\mathrm{S}$ exportadas pelos grãos (Figura 2) não foram alteradas pelas doses desse nutriente aplicadas, mas refletiram, na grande maioria dos casos, a sua disponibilidade no solo nos locais com maior exportação desse nutriente, onde os teores eram maiores (13,4 a 16,5 $\mathrm{mg} \mathrm{dm}^{-3}$ ) em relação aos demais. No local com maior exportação (16,5 $\left.\mathrm{mg} \mathrm{dm}^{-3} \mathrm{de} \mathrm{S}\right)$, a média foi de 4,12 $\mathrm{kg} \mathrm{ha}^{-1} \mathrm{de} \mathrm{S}$. Considerando-se que o rendimento médio de grãos foi de aproximadamente 9,0 $\mathrm{tha}^{-1}$, essa quantidade exportada fica abaixo daquela estimada por Wilson Jr. et al. (2006), em Arkansas - EUA, que era de $7 \mathrm{~kg} \mathrm{ha}^{-1}$ em lavouras de rendimento próximo a 7,0 $\mathrm{t} \mathrm{ha}^{-1}$, ou seja, $1 \mathrm{~kg}$ de $\mathrm{S}$ exportado por t de grãos produzida. Essas diferenças podem, no entanto, ocorrer por diversos fatores, como diferenças de clima e solo, mas principalmente varietais, em especial no que se refere ao seu ciclo, o que interfere na quantidade de nutrientes absorvidos ao longo do período vegetativo. Variedades de ciclo precoce, como as utilizadas neste estudo, têm período vegetativo de cerca de 60 dias, enquanto em variedades de ciclo tardio esse período é de aproximadamente 90 dias, ou mais (Wilson Jr. et al., 2006).

\section{Calibração do teor de enxofre no solo e recomendações de adubação}

A curva de calibração do S (Figura 3) foi obtida pela utilização dos dados de rendimento de arroz das parcelas sem a adição de $\mathrm{S}$ ao solo, em relação ao rendimento máximo obtido em cada local de condução dos experimentos, nos dois anos agrícolas (2004/2005 

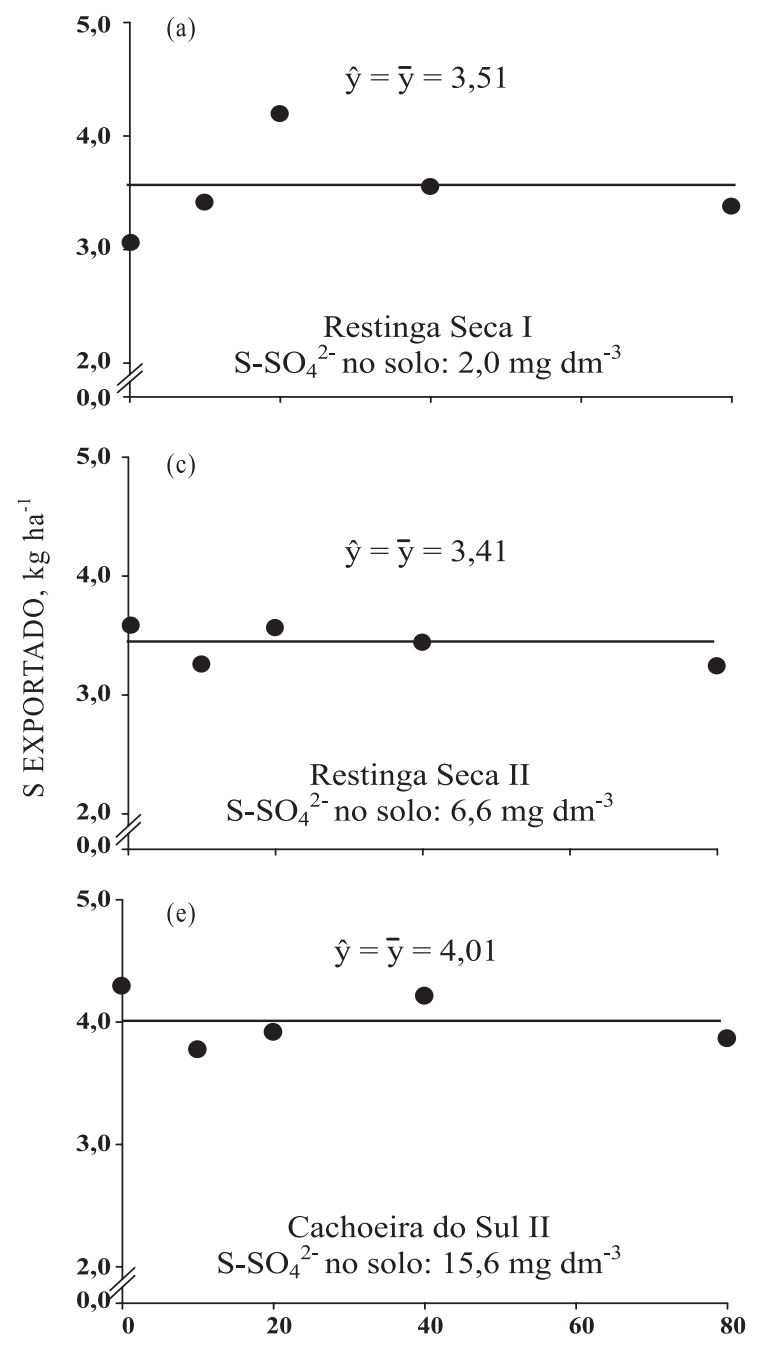

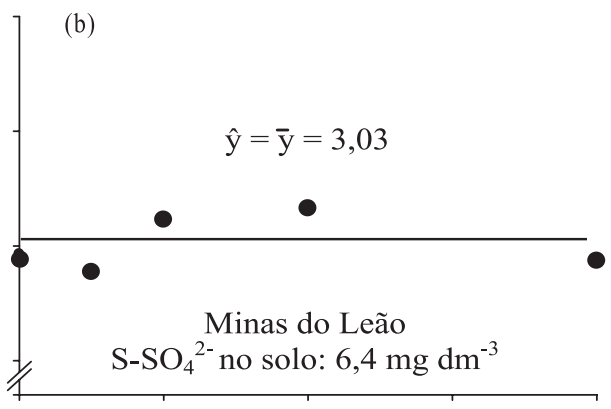

(d)

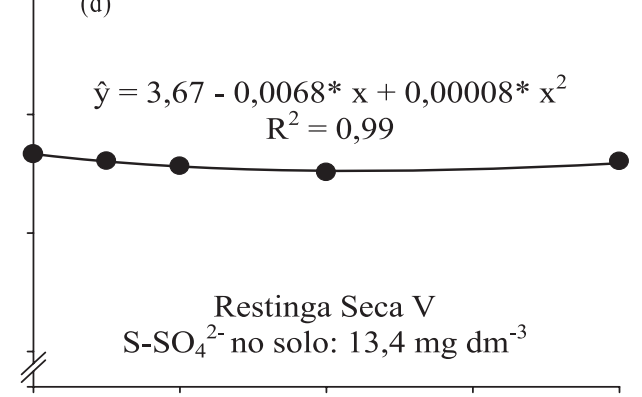

(f)

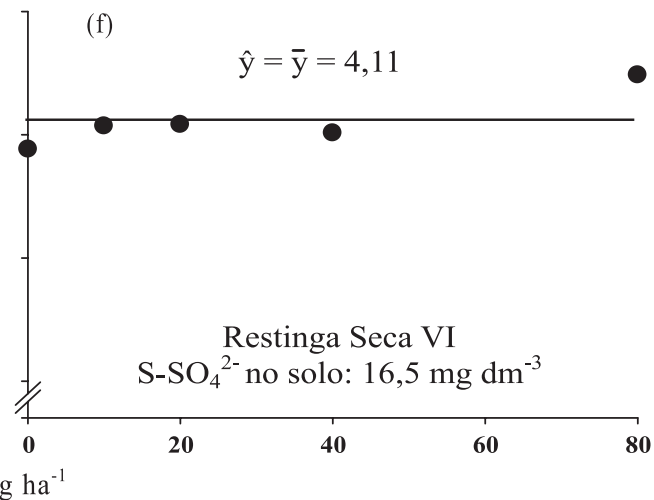

Figura 2. Enxofre exportado pelo arroz irrigado, em função da aplicação de doses deste elemento em diferentes locais, na safra $2005 / 2006$. *Significativo pelo teste $\mathrm{F}(\mathrm{p}<0,05)$.

e 2005/2006), em função do teor inicial de S no solo (Quadro 1). O teor crítico foi determinado pelo procedimento descrito por Cate \& Nelson (1965), que consiste em enquadrar o maior número possível de pontos nos quadrantes positivos. Isso, aplicado aos resultados da figura 3 , resultou na definição de um valor crítico próximo de $9,0 \mathrm{mg} \mathrm{dm}^{-3} \mathrm{de} \mathrm{S}$, o que corresponde a aproximadamente $95 \%$ do rendimento máximo. Esse valor é superior ao teor crítico que vinha sendo utilizado no Rio Grande do Sul e em Santa Catarina em culturas pouco exigentes no nutriente, incluindo o arroz irrigado, que é de $5,0 \mathrm{mg} \mathrm{dm}^{-3}$ e próximo de $10,0 \mathrm{mg} \mathrm{dm}^{-3}$ naquelas que exigem mais (CQFS RS/SC, 2004). A resposta à adição de S ao solo foi sempre positiva nos solos com teor de $\mathrm{S}$ inferior ao nível crítico estabelecido.

Neue \& Mamaril (1984), revisando trabalhos com o método de extração por solução de $\mathrm{Ca}\left(\mathrm{H}_{2} \mathrm{PO}_{4}\right)_{2}$, utilizado neste trabalho, chegaram a teores críticos

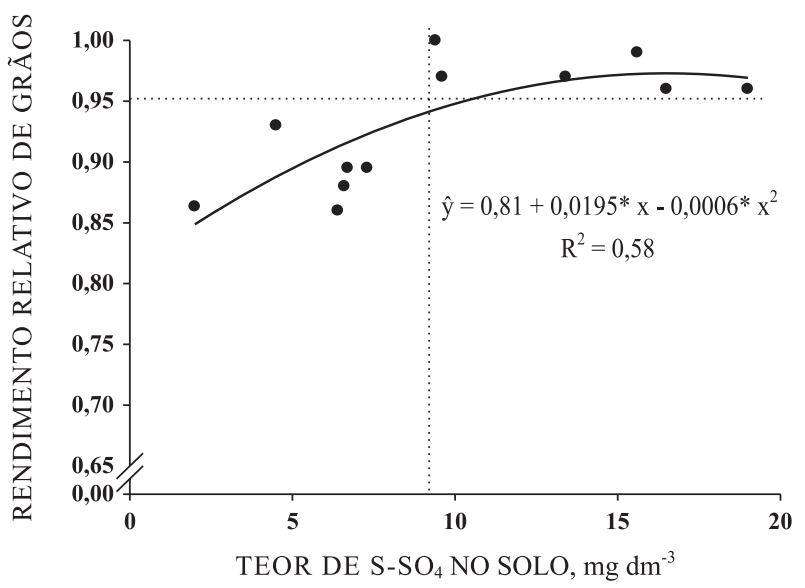

Figura 3. Curva de calibração de enxofre de solos da região da Depressão Central do RS. Safras 2004/ 2005 e 2005/2006. *: significativo pelo teste $F$ $(\mathrm{p}<0,05)$. 
para o arroz irrigado entre 7,0 e $11,0 \mathrm{mg} \mathrm{dm}^{-3}$, resultando em uma média de $9,0 \mathrm{mg} \mathrm{dm}^{-3}$, o mesmo valor determinado neste trabalho, porém menor do que o teor encontrado em solos da Colômbia, de $12 \mathrm{mg} \mathrm{dm}^{-3}$ (Riobueno, 2003).

A partir da obtenção da curva de calibração e do teor crítico, foram estabelecidas as faixas de teores para a interpretação dos resultados das análises de solo (baixo, médio e alto) e elaboração das recomendações de adubação, com base na resposta da cultura à adição de doses crescentes de $\mathrm{S}$, em cada faixa de interpretação. Adotou-se o teor crítico de $10 \mathrm{mg} \mathrm{dm}^{-3}$ de $\mathrm{S}$ no solo, visando facilitar a subdivisão em três faixas de interpretação: 0-5,0 - baixo; 5,1-10,0 - médio; e > 10,0 mg dm ${ }^{-3}$ - alto (SOSBAI, 2007). Essa tentativa não teve sucesso, uma vez que não houve distinção de resposta do arroz entre as faixas baixo (0-5,0 $\left.\mathrm{mg} \mathrm{dm}^{-3}\right)$ e médio $\left(5,1-10,0 \mathrm{mg} \mathrm{dm}^{-3}\right)$. A alternativa foi, então, estabelecer a resposta média da cultura em somente duas faixas: abaixo e acima do teor crítico.

A resposta do arroz foi diferenciada em cada situação: na faixa abaixo do teor crítico (baixo), houve uma resposta quadrática ascendente $\left(\mathrm{R}^{2}=0,95\right)$ (Figura 4a) com ajuste significativo ( $p<0,05)$, e na faixa acima do teor crítico (alto), não houve resposta (Figura 4b). Assim, em solos com teor inferior ao crítico estabelecido (Figura 4a), o aumento no rendimento de grãos ocorre, de fato, até a aplicação entre 20 e $40 \mathrm{~kg} \mathrm{ha}^{-1}$ de $\mathrm{S}$, um pouco acima do que indicam os trabalhos de Beaton \& White (1997) e Wilson Jr. et al. (2006), que estabeleceram a recomendação de $20 \mathrm{~kg} \mathrm{ha}^{-1}$ de $\mathrm{S}$ em solos deficientes nesse nutriente. Pelos dados deste trabalho, em situações de aplicação desse nutriente em solos com teor acima do crítico, não haverá resposta à aplicação de S.

Havlin et al. (2005) afirmam que a dose de máxima eficiência econômica (DMEE) do nutriente, ou seja, a dose a ser recomendada está relacionada a uma produtividade entre 80 e $95 \%$ do rendimento máximo da cultura. O cálculo dessa dose é, no entanto, definido pelo preço do insumo (dose do adubo contendo S) e do produto - no caso, o arroz irrigado - e pode ser efetuado a partir da função de produção para teores de S no solo abaixo do crítico $\left(<10,0 \mathrm{mg} \mathrm{dm}^{-3}\right)$. Neste trabalho, inicialmente foi calculada a dose de máxima eficiência técnica (DMET), a partir da respectiva função de produção (Figura 4a), que correspondeu a $36 \mathrm{~kg} \mathrm{ha}^{-1} \mathrm{de}$ S.

Considerando-se a DMET obtida e a dose de N aplicada nos tratamentos nas safras 2004/2005 (210 kg ha-1 de N) e 2005/2006 (160 kg ha-1 de N), há, respectivamente, uma relação de cerca de seis e quatro partes de N para uma de S. Bixby \& Beaton (1970) consideram que, em solos onde esses dois nutrientes são limitantes, a relação N:S aplicada deve ser de 7:1.

Substituindo o valor da DMET na equação da função de produção, chega-se ao rendimento de $7,51 \mathrm{t} \mathrm{ha}^{-1}$, que representa um ganho de $0,39 \mathrm{t} \mathrm{ha}^{-1}$ em relação à testemunha, um valor menor do que o ganho máximo médio observado de 1,00 $\mathrm{t} \mathrm{ha}^{-1}$.

Para o cálculo da dose de máxima eficiência econômica (DMEE) de S (X), leva-se em consideração o preço de venda do arroz $(\mathrm{V})$, o rendimento de grãos (Y), o custo do $\mathrm{S}\left(\mathrm{F}_{1}\right)$ e os custos fixos (Fo). Logo, o lucro líquido $(\mathrm{L})$ corresponde a:

$$
\mathrm{L}=\mathrm{VY}-\mathrm{Fo}-\mathrm{F}_{1} \mathrm{X}
$$

Em pesquisa de mercado realizada no momento dos cálculos, o preço do arroz tipo 1 Longo Fino (Agrolink, 2007) e o do S eram de, respectivamente, $\mathrm{R} \$ 387,00 \mathrm{t}^{-1}$ e $\mathrm{R} \$ 2.170,00 \mathrm{t}^{-1}$, ou $\mathrm{R} \$ 0,39 \mathrm{~kg}^{-1} \mathrm{e}$ $\mathrm{R} \$ 2,17 \mathrm{~kg}^{-1}$. A partir desses dados e considerando-se os preços do quilo de $\mathrm{S}$ e de arroz, foi determinada a dose de máxima eficiência econômica (DMEE), conforme Grimm (1970), de $26 \mathrm{~kg} \mathrm{ha}^{-1}$ de S, um valor de $10 \mathrm{~kg} \mathrm{ha}^{-1}$ de $\mathrm{S}$ inferior à DMET e que corresponde a um rendimento de $7,48 \mathrm{tha}^{-1}$. Verifica-se, pois, que

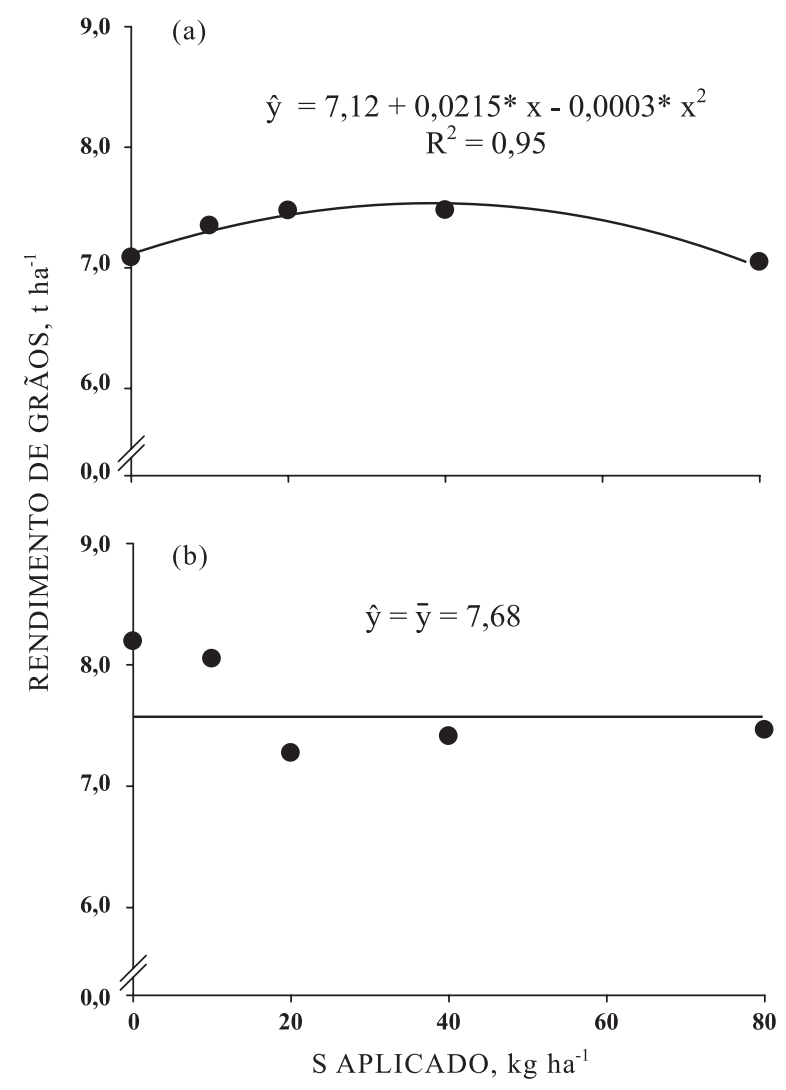

Figura 4. Rendimento de arroz irrigado, em função de doses de $\mathrm{S}$, em solos com teores de $\mathrm{S}_{-} \mathrm{SO}_{4}{ }^{2-}$ abaixo do crítico (a) e acima do crítico (b). Safras 2004/2005 e 2005/2006. *: significativo pelo teste F $(\mathbf{p}<0,05)$. 
a DMET e a DMEE proporcionam rendimentos de grãos semelhantes.

Em se tratando do custo adicional que a DMEE implica para o suprimento desse nutriente à cultura do arroz irrigado, chega-se a um valor de $R \$ 18,40 \mathrm{ha}^{-1}$, ou seja, um dispêndio muito baixo, se for levado em conta que o rendimento adicional médio obtido, de $0,36 \mathrm{tha}^{-1}$, gera um lucro líquido adicional de $\mathrm{R} \$ 122,88 \mathrm{ha}^{-1}$, pois o sulfato de amônio, por conter $21 \%$ de $\mathrm{N}$ em sua formulação, tem seu custo abatido pela adição de menor quantidade de ureia $\left(\mathrm{R} \$ 740,00 \mathrm{t}^{-1}\right.$ Pesquisa de Mercado, 2007), visto que a aplicação de $110 \mathrm{~kg} \mathrm{ha}^{-1}$ de $\left(\mathrm{NH}_{4}\right)_{2} \mathrm{SO}_{4}$, além de aportar ao cultivo a DMEE de $26 \mathrm{~kg} \mathrm{ha}^{-1}$ de $\mathrm{S}$, também disponibiliza $23 \mathrm{~kg} \mathrm{ha}^{-1}$ de N.

\section{CONCLUSÕES}

1. Os teores disponíveis de $\mathrm{S}$ nas amostras de solo não se relacionam com os teores de matéria orgânica e de argila.

2. Os teores de S no tecido e a sua absorção pelo arroz aumentam com a sua disponibilidade no solo e com as doses de adubo aplicadas somente em solos com os teores de $\mathrm{S}$ mais baixos $\left(\leq 6,4 \mathrm{mg} \mathrm{dm}^{-3}\right)$.

3. O teor de $\mathrm{S}$ nas plantas de arroz não atinge a faixa de suficiência pela interpretação do seu estado nutricional em uso no Brasil, mesmo com produtividade de grãos entre 7,0 e 9,0 t ha-1.

4. O nível crítico de $\mathrm{S}$ determinado para solos da Depressão Central do Rio Grande do Sul $\left(9 \mathrm{mg} \mathrm{dm}^{-3}\right)$ é superior ao utilizado no sul do Brasil $\left(5 \mathrm{mg} \mathrm{dm}^{-3}\right)$.

5. As doses de máxima eficiência técnica $\left(36 \mathrm{~kg} \mathrm{ha}^{-1}\right)$ e econômica $\left(26 \mathrm{~kg} \mathrm{ha}^{-1}\right)$ para o arroz irrigado em solos com teor abaixo do crítico resultam em um ganho similar no rendimento de grãos.

\section{LITERATURA CITADA}

AGROLINK. Disponível em: <http://www.agrolink.com.br/ cotacoes/index.asp>. Acesso em: 15 jan. 2007.

ALAM, M.L.; AHMED, F. \& KARIM, M. Effect of different sources of sulphur on rice under submerged condition. J. Ind. Soc. Soil Sci., 33:586-590, 1985.

BEATON, J.D. \& WHITE, M. Occurrence and correction of S deficiencies in the Asian and Pacific Region: A review and update. Sulphur Agric., 20:31-46, 1997.

BISSANI, C.A. Disponibilidade de enxofre para as plantas em solos do Rio Grande do Sul. Porto Alegre, Universidade Federal do Rio Grande do Sul, 1985. 198p. (Tese de Mestrado)
BISSANI, C.A.; CAMARGO, F.A.O.; GAINELLO, C. \& TEDESCO, M.J. Fertilidade dos solos e manejo da adubação de culturas. 2.ed. Porto Alegre, Metrópole, 2008. $344 \mathrm{p}$.

BIXBY, D.W. \& BEATON, J.D. Sulphur containing fertilizers, properties and applications. Washington, The Sulphur Institute, 1970. 27p. (Technical Bulletin, 17)

CARMONA, F.C. Enxofre para o arroz irrigado em solos da Depressão Central do Rio Grande do Sul. Porto Alegre, Universidade Federal do Rio Grande do Sul, 2007. 70p. (Tese de Mestrado)

CATE, R.B. \& NELSON, L.A. Um método rápido para correlação de análise de solo com ensaios de adubação. Raleigh, North Carolina State University, 1965. (Boletim Técnico, 1)

CHAITEP, W.; LEFROY, R.D.B. \& BLAIR, G.J. Effect of placement and source of sulfur in flooded and non-flooded rice cropping systems. Austr. J. Agric. Res., 45:1547-1556, 1994.

COMISSÃO DE QUÍMICA E FERTILIDADE DO SOLO CQFS RS/SC. Manual de recomendações de adubação e calagem para os Estados do Rio Grande do Sul e Santa Catarina. 10.ed. Porto Alegre, SBCS/NRS, 2004. 400p.

CONAB. Disponível em: <http://www.conab.gov.br/conabweb/ download/safra/6levsafra.pdf>. Acesso em: 6 jul. 2007.

COUNCE, P.A.; KEISLING, T.C. \& MITCHELL, A. A uniform, objective, and adaptive system for expressing rice development. Crop Sci., 40:436-443, 2000.

EMPRESA BRASILEIRA DE PESQUISA AGROPECUÁRIA EMBRAPA. Sistema brasileiro de classificação de solos. Brasília, Embrapa Produção de Informação, 1999. 412p.

ENSMINGER, L.E. Some factors affecting the adsorption of sulfate by Alabama soils. Soil Sci. Soc. Am. Proc., 18:259264,1954

GRIMM, S.S. Aspectos econômicos da adubação. Porto Alegre, Universidade Federal do Rio Grande do Sul, 1970. 14p. (Boletim Técnico, 6)

GUINDANI, R.H.; ANGUINONI, I. \& NACHTIGALL, G.R. DRIS na avaliação do estado nutricional do arroz irrigado por inundação. R. Bras. Ci. Solo, 33:109-118, 2009.

HAVLIN, J.L.; BEATON, J.D.; TISDALE, S.L. \& NELSON, W.L. Soil fertility and fertilizers: An introduction to nutrient management. 7.ed. New Jersey, Pearson Prentice Hall, 2005. 515p.

HINGSTON, R.J.; POSNER, A.M. \& QUIRK, J.P. Anion adsorption by goethite and gibbsite I. The role of the proton in determining adsorption envelopes. J. Soil Sci., 23:177192, 1972.

IRRI. Disponível em: <http://www.knowledgebank.irri.org/ ricedoctor_mx/Fact_Sheets/DeficienciesToxicities/ Sulfur_Deficiency.htm> Acesso em: 20 jan. 2007.

IRGA. Disponível em: <http://www.irga.rs.gov.br/arquivos/ 20060524160029.pdf.> Acesso em: 17 fev. 2008.

JORDAN, H.V. \& ENSMINGER, L.E. The role of sulfur in soil fertility. Adv. Agron., 10:407-434, 1958. 
LEFROY, R.D.B.; MAMARIL, C.P.; BLAIR, G.J. \& GONZALES, P.J. Disponível em: <http://www.icsn-scope.org/ downloadpubs/scope48/chapter11.html\#t2>. Acesso em: 16 set. 2005.

MALAVOLTA, E.; VITTI, G.C. \& OLIVEIRA, S.A. Avaliação do estado nutricional das plantas: Princípios e aplicações. 2.ed. Piracicaba, Potafos, 1997. 319p.

MANDAL, B.K.; CHATTERJEE, B.N. \& MUKHOPADHYAY, P. Direct and residual effects of different $\mathrm{S}$ fertilizers in rice-based sequential cropping in West Bengal, India. Sulphur Agric., 20:47-53, 1997.

MARCOLIN, E.; MACEDO, V.R.M. \& GENRO JUNIOR, S.A. Rendimento de grãos, perdas de solo e de nutrientes em função de manejos do solo, da adubação e da água no sistema pré-germinado. In: CONGRESSO BRASILEIRO DE ARROZ IRRIGADO, 4.; REUNIÃO DA CULTURA DO ARROZ IRRIGADO, 26., Santa Maria, 2005. Anais. Santa Maria, Sociedade Sul Brasileira de Arroz Irrigado, 2005. v.1. p.350-353.

MEDINA, A.C. Mejore los rendimientos y la calidad del arroz aplicando azufre. R. Arroz, 51:56-58, 2003.

NEUE, H.E. \& MAMARIL, C.P. Zinc, sulfur and other micronutrients in wetland soils. In: WETLAND SOILS CHARACTERIZATION, CLASSIFICATION AND UTILIZATION. Los Baños, 1984. Los Baños, International Rice Research Institute, 1984. p.307-319

OSÓRIO FILHO, B.D.; RHEINHEIMER, D.S.; SILVA, L.S.; KAMINSKI, J. \& DIAS, G.F. Deposição do enxofre atmosférico no solo pelas precipitações pluviais e respostas de culturas à adubação sulfatada em sistema plantio direto. Ci. Rural, 37:712-719, 2007.

PINTO, L.F.S.; LAUS NETO, J.A. \& PAULETTO, E.A. Solos de várzea do Sul do Brasil cultivados com arroz irrigado. In: GOMES, A.S. \& MAGALHÃES, A.M. Arroz Irrigado no Sul do Brasil. Brasília, Embrapa, 2004. p.75-96.
PULVER, E.; CARMONA, L.C. \& CARMONA, F.C. Tratamento de sementes como estratégia de manejo para altas produtividades. In: CONGRESSO BRASILEIRO DE ARROZ IRRIGADO, 4.; REUNIÃO DA CULTURA DO ARROZ IRRIGADO, 26., Santa Maria, 2005. Anais. Santa Maria, Sociedade Sul Brasileira de Arroz Irrigado, 2005. v.2. p.129-131.

PULVER, E. \& CARMONA, L.C. Reduzindo as lacunas de produtividade em arroz irrigado na Venezuela e Rio Grande do Sul, Brasil. Cachoeirinha, IRGA, 2004. 54p.

RIOBUENO, C.M. Respuesta de FEDEARROZ 2000 a la fertilización con azufre. R. Arroz, 51:85-87, 2003.

STRECK, E.D.; KAMPF, N.; DALMOLIN, R.S.D.; KLAMT, E.; NASCIMENTO, P.C.; SCHNEIDER, P.; GIASSON, E. \& PINTO, L.F.S. Solos do Rio Grande do Sul. 2.ed. Porto Alegre, EMATER/RS-ASCAR, 2008. 222p.

SOCIEDADE SUL-BRASILEIRA DE ARROZ IRRIGADO SOSBAI. Recomendações técnicas da pesquisa para o Sul do Brasil. Santa Maria, 2005.159p.

SOCIEDADE SUL-BRASILEIRA DE ARROZ IRRIGADO SOSBAI. Recomendações técnicas da pesquisa para o Sul do Brasil. Pelotas, 2007. 161p.

TEDESCO M.J.; GIANELLO, C.; BISSANI, C.A.; BOHNEM, H. \& VOLKWEISS, S.J. Análise de solo, plantas e outros materiais. 2.ed. Porto Alegre, Universidade Federal do Rio Grande do Sul, 1995. 188p. (Boletim Técnico, 5)

VITTI, G.C.; FERREIRA, M.E. \& MALAVOLTA, E. Respostas de culturas anuais e perenes ao fosfogesso. In: SEMINÁRIO SOBRE O USO DE FOSFOGESSO NA AGRICULTURA, 1.; Brasília, 1985. Anais. Brasília, Empresa Brasileira de Pesquisa Agropecuária, 1986. p.17-44.

WILSON JR., C.; SLATON, N.; NORMAN, R. \& MILLER, D. Rice production handbook. Arkansas, Cooperative Extension Service University of Arkansas, 2006. 126p. 